\title{
Evidence for a shear velocity discontinuity in the lower mantle beneath India and the Indian Ocean
}

\author{
Christopher J. Young and Thorne Lay \\ Department of Geological Sciences, University of Michigan, Ann Arbor, MI 48109 (U.S.A.)
}

(Received and accepted December 18, 1986)

\begin{abstract}
Young, C.J. and Lay, T., 1987. Evidence for a shear discontinuity in the lower mantle beneath India and the Indian Ocean. Phys. Earth Planet. Inter., 49: 37-53.

SH and sSH seismograms are modeled to determine the shear velocity structure in the $\mathrm{D}^{\prime \prime}$ region beneath India and the Indian Ocean. The signals show waveform complexities similar to those observed in data sampling the $\mathrm{D}^{\prime \prime}$ region beneath Alaska, the Caribbean, and Eurasia (Lay and Helmberger), which have been attributed to a $2.7 \%$ shear velocity discontinuity $-280 \mathrm{~km}$ above the core-mantle boundary. The new data set consists of long-period tangential component recordings at WWSSN stations in Africa, the Middle East, and Europe for 11 intermediate and deep focus Indonesian earthquakes. In the distance range $70-82^{\circ}$ the waveforms show an arrival between $\mathrm{SH}$ and $\mathrm{ScSH}$ with systematic moveout. From 89 to $94^{\circ}$ there is a strong distortion of the SH waveforms, indicating the arrival of several phases closely spaced in time. The relative time shifts of similar complexity in the corresponding sSH phases requires a deep mantle origin. The depth dependence and moveout of the interference effects are well-predicted for both SH and $\mathrm{sSH}$ phases by a model with a lower mantle discontinuity. Alternative explanations of the interference as resulting from receiver reverberations, SKS contamination, multiple source complexity, or near source multipathing are ruled out by systematic tests. While it is apparent that lateral variations in the lower mantle velocity structure prevent any single model from fitting all of the data, synthetic waveform modeling (using generalized ray theory and reflectivity) shows that the data can be well-fit by a model with a discontinuity similar in size and depth to that proposed for the previously investigated regions (Lay and Helmberger), but with a negative velocity gradient within the $\mathrm{D}^{\prime \prime}$ layer.
\end{abstract}

\section{Introduction}

The velocity structure of the lowermost $200 \mathrm{~km}$ of the mantle, designated $D^{\prime \prime}$ by Bullen (1949), has been a controversial topic ever since Gutenberg and Richter (1939) and Jeffreys (1939) proposed that the velocity gradients just above the coremantle boundary (CMB) are anomalously low. Many subsequent seismological models for this region have been advanced, sharing the common feature that the $D^{\prime \prime}$ region is distinct from the rest of the lower mantle, but differing markedly in detail (see Young and Lay (1987) for a review). Most contemporary velocity models for the lowermost mantle have either slightly positive or slightly negative velocity gradients in $\mathrm{D}^{\prime \prime}$, with smooth overall velocity variations. These gradients are diminished from the positive gradients in the overlying mantle, indicating a departure from homogeneous structure. However, these smooth models cannot explain many features of the seismic observations, and caution is required in interpreting them, even as 'average' velocity structures for the region.

The disagreement about the velocity structure of $\mathrm{D}^{\prime \prime}$ is probably principally due to the strong velocity heterogeneity in the region, occurring on scales ranging from tens to thousands of kilometers (Alexander and Phinney, 1966; Cleary and Haddon, 1972; Doornbos and Vlaar, 1973; Haddon and Cleary, 1974; King et al., 1974; Wright, 1975; Husebye et al., 1976; Bolt and Niazi, 1984). 
In the last decade the accumulation of large sets of travel times has made it possible to perform inversions for long-wavelength features of the three-dimensional velocity structure of the deep Earth (Sengupta and Toksoz, 1976; Dziewonski et al., 1977, Sengupta et al., 1981; Clayton and Comer, 1983; Dziewonski, 1984). These inversions, while insensitive to fine scale features in $\mathrm{D}^{\prime \prime}$, do indicate that this region has a greater degree of heterogeneity than any other part of the lower mantle. Even in the presence of this largescale lateral heterogeneity, radial velocity models derived from data that are sensitive to fine structure in $\mathrm{D}^{\prime \prime}$ are still useful for analyzing the structure and dynamics of the lower mantle, as long as such models are developed for localized regions over which the lateral velocity variations may be slowly varying.

There is a growing body of evidence suggesting that the velocity structure of the lowermost mantle does not have smooth velocity gradients in all locations. If any $\mathbf{P}$ or $\mathbf{S}$ wave velocity discontinuities are present in $D^{\prime \prime}$ they will produce triplications in the $\mathbf{P}$ and $\mathrm{S}$ travel-time curves between 70 and $95^{\circ}$. A series of studies using short-period $P$ data from arrays in Canada and Australia (Wright, 1973; Wright and Lyons, 1975, 1980/1981; Wright et al., 1985), have suggested the presence of a triplication in the $\mathrm{P}$ travel-time curve resulting from an abrupt $1.5-3.0 \% \mathrm{P}$ velocity discontinuity $\sim 200 \mathrm{~km}$ above the CMB. These studies argue for the presence of the triplication on the basis of careful measurements of the change in slowness of $P$ waves with distance and subtle waveform interference patterns, procedures which require accurate corrections for source and receiver effects. A sharp change in the slope of the first arrival branch of $\mathrm{P}$ near $85^{\circ}$ is believed to represent the crossing over of the back branch of the triplication (Wright et al., 1985). The high noise level of typical shortperiod $\mathbf{P}$ coda makes identification of later arriving branches of the proposed triplication difficult, and detailed waveform modeling of the array data has not been performed.

Lay and Helmberger (1983) presented evidence for an $\mathrm{S}$ wave triplication, manifested by an unexpected arrival between $\mathrm{SH}$ and $\mathrm{ScSH}$ in the distance range $70-85^{\circ}$ and by distortion of the $\mathrm{SH}$ waveforms in the range $88-92^{\circ}$. Because shear velocities in $\mathrm{D}^{\prime \prime}$ are much slower than corresponding $P$ velocities, the branches of an $S$ wave triplication are more separated than the branches of a corresponding $\mathrm{P}$ wave triplication; hence, it is easier to identify secondary $\mathbf{S}$ arrivals. Lay and Helmberger (1983) performed forward modeling that directly compared long-period SH data with

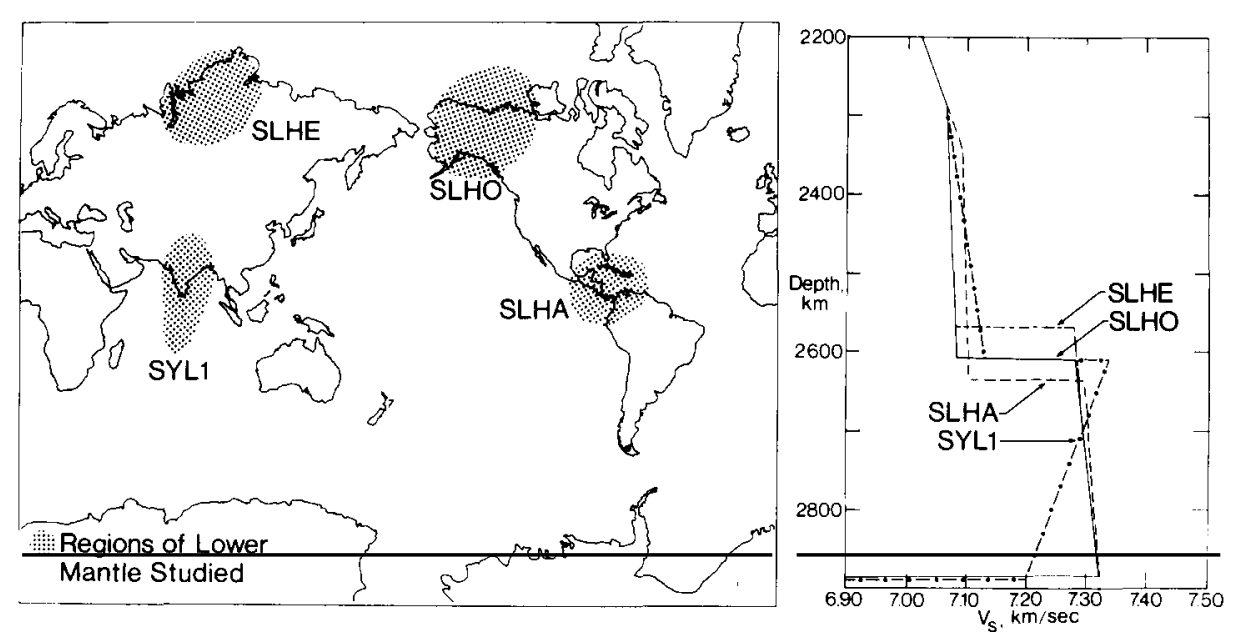

Fig. 1. (Left) The four regions of the lower mantle for which shear velocity discontinuities have been proposed. (Right) Velocity vs. depth profiles for the four proposed discontinuity models. SLHA, SLHE and SLHO represent modifications of the JB model; SYL1 is a modification of the PREM model. 
synthetic waveforms to determine that a $2.75 \% \mathrm{~S}$ velocity discontinuity $\sim 280 \mathrm{~km}$ above the core could explain the systematic travel time and amplitude behavior of the extra arrivals. Although their discontinuity model was shown to fit extensive data sets for regions beneath Alaska, the Caribbean, and northern Eurasia (Fig. 1), it is unable to fit some data at diffracted distances (beyond $95^{\circ}$ ) as well as do smooth velocity models (Schlittenhardt et al., 1985).

In this study, a new shear velocity discontinuity model, SYL1, is proposed for the $\mathrm{D}^{\prime \prime}$ region beneath India and the Indian Ocean (Fig. 1). This model is obtained by waveform modeling (generalized ray theory and reflectivity) using the Preliminary Reference Earth Model (PREM) velocity structure of Dziewonski and Anderson (1981) as a smooth starting model. The final model is similar to the previously proposed $\mathrm{S}$ discontinuity models for other regions in the size (2.8\%) and depth (280 $\mathrm{km}$ above the core-mantle boundary) of the discontinuity, but it has a negative velocity gradient below the discontinuity rather than the mildly positive one in the Lay and Helmberger (1983) models. This negative gradient makes it possible for model SYL1 to fit SH waveforms beyond $95^{\circ}$ better than a smooth velocity structure, while still fitting data in the $70-95^{\circ}$ range. Lay (1985) has shown that similar modifications of the structures proposed for other regions can eliminate the objections about diffracted effects for $\mathrm{S}$ waves raised by Schlittenhardt et al. (1985).

\section{Data set}

The data used in this study are from 11 earthquakes in the Java Trench-Timor Trough region which were recorded at 21 long-period WWSSN stations in Africa, the Middle East, and Europe (Fig. 2, Table I). These events are associated with the subduction of the Australian plate beneath the Southeast Asian plate, along a trench with a nearly east-west strike (Cardwell and Isacks, 1978). The location of the stations relative to the subducted slab (Fig. 2) makes some multipathing or diffraction effects due to the high velocity slab likely (Silver and Chan, 1987), but these processes are unlikely to produce the systematic changes in waveform with distance, which we will show to be

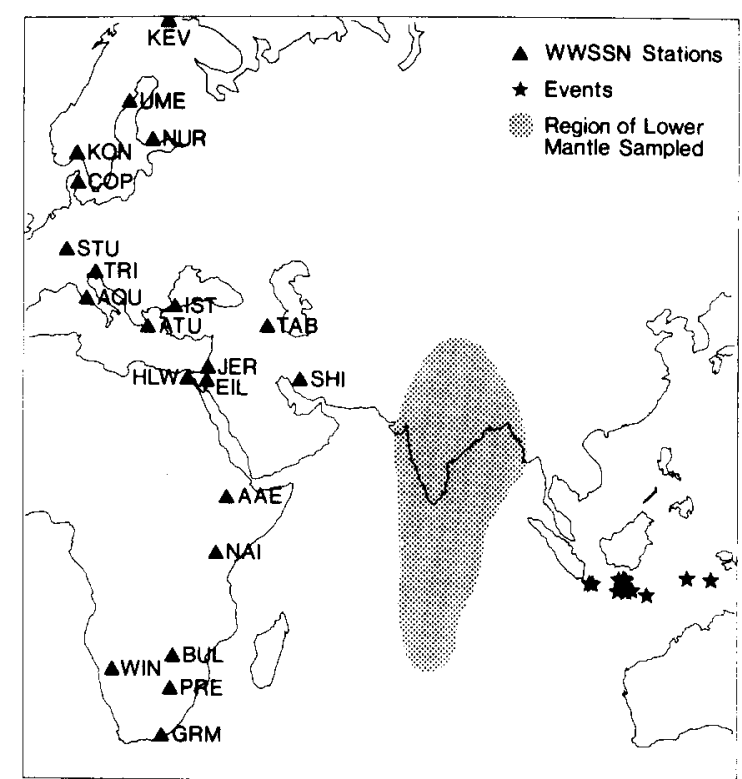

Fig. 2. The 21 WWSSN stations and 11 Indonesian events used in this study. The stippled region indicates the portion of $D^{\prime \prime}$ sampled by the data.

characteristic of our data. The selected events have source depths ranging from 80 to $636 \mathrm{~km}$, moderate magnitudes $\left(m_{\mathrm{b}}=5.7-6.2\right)$, and strong $\mathrm{SH}$ radiation to the stations. The events were chosen for their simple impulsive waveshapes that indicate minimal complexity from source rupture processes. The long-period $\mathrm{S}$ waveforms were digitized and rotated to obtain the SH components, although many of the stations are close to being naturally rotated for these paths. The SV waveforms were not used in this study because they contain the additional SKS phase, which arrives between $\mathrm{S}$ and $\mathrm{ScS}$ at distances $<82^{\circ}$, and can thus obscure any triplication arrivals. Contamination of the SH components by SKS energy can occur if there is any rotation of the S polarization vector along the raypath, but it has been shown that SKS contamination is typically minor for these long-period signals when the SH radiation from the source is strong (Lay and Young, 1986).

The large range in source to receiver azimuth spanned for each event in this study (a total of about $85^{\circ}$ ) predicts significant relative amplitude 
TABLE I

Source parameters for events used in this study

\begin{tabular}{|c|c|c|c|c|c|c|c|}
\hline Date & & Time & Lat. & Long. & Depth (km) & $M_{\mathrm{b}}$ & Reference \\
\hline 24 Mar. & 1967 & $09: 00: 19.1$ & $5.9^{\circ} \mathrm{S}$ & $112.3^{\circ} \mathrm{E}$ & 595 & 5.7 & USCGS \\
\hline 13 Apr. & 1969 & $23: 33: 15.4$ & $6.1^{\circ} \mathrm{S}$ & $129.9^{\circ} \mathrm{E}$ & 152 & 5.9 & USCGS \\
\hline 04 Aug. & 1969 & $17: 19: 19.6$ & $5.7^{\circ} \mathrm{S}$ & $125.3^{\circ} \mathrm{E}$ & 521 & 6.2 & USCGS \\
\hline 13 Feb. & 1970 & $15: 43: 28.7$ & $5.9^{\circ} \mathrm{S}$ & $113.0^{\circ} \mathbf{E}$ & 636 & 5.8 & USCGS \\
\hline 13 Aug. & 1970 & $04: 22: 38.5$ & $8.9^{\circ} \mathrm{S}$ & $118.0^{\circ} \mathrm{E}$ & 117 & 6.0 & USCGS \\
\hline 04 Nov. & 1972 & $21: 36: 01.7$ & $8.2^{\circ} \mathrm{S}$ & $112.3^{\circ} \mathrm{E}$ & 126 & 6.0 & NEIS \\
\hline $17 \mathrm{May}$ & 1974 & $20: 55: 11.2$ & $6.5^{\circ} \mathrm{S}$ & $106.8^{\circ} \mathrm{E}$ & $126^{a}$ & 6.0 & NEIS \\
\hline $28 \mathrm{Dec}$ & 1975 & $15: 24: 50.8$ & $8.0^{\circ} \mathrm{S}$ & $115.1^{\circ} \mathrm{E}$ & 196 & 5.9 & NEIS \\
\hline 01 Jan. & 1977 & $17: 35: 54.9$ & $7.9^{\circ} \mathrm{S}$ & $109.0^{\circ} \mathrm{E}$ & $80^{a}$ & 5.7 & NEIS \\
\hline 07 May & 1979 & $12: 52: 06.3$ & $6.3^{\circ} \mathrm{S}$ & $106.0^{\circ} \mathrm{E}$ & $106^{a}$ & 5.9 & NEIS \\
\hline $09 \mathrm{Jul}$. & 1984 & $23: 19: 03.5$ & $5.8^{\circ} \mathrm{S}$ & $111.3^{\circ} \mathrm{E}$ & 534 & 5.8 & NEIS \\
\hline
\end{tabular}

a Depths redetermined based on $\mathrm{SS}-\mathrm{S}$ times.

changes of $\mathrm{SH}$ phases due to the $\mathrm{SH}$ radiation patterns of the sources. This was less of a problem in the study by Lay and Helmberger (1983), where the dense distribution of North American stations allowed the use of station profiles in a fairly narrow azimuthal rainge. To account for the radiation pattern effects in the data, we have included appropriate focal mechanisms in the calculation of the SH and sSH synthetics (see Helmberger (1974) for a discussion of this technique). The focal mechanisms (Table II) for the five events with profiles of data shown in this study were taken from the literature or newly determined by analysis of $\mathrm{P}$ wave first motions and $\mathrm{S}$ polarizations (Dillinger et al., 1972).

A composite travel-time plot of the measured tangential component $\mathbf{S}$ travel times from the 11 events in our data set is shown in Fig. 3. Arrival times for $\mathbf{S}, \mathrm{ScS}$, and an intermediate arrival (which was consistently observed) are shown. The travel times and distances have been corrected to corre- spond to a surface focus source by raytracing from the actual source depths to the surface, and averaged station JB travel-time residuals have been subtracted. Only data from stations with four or more travel-time measurements (including $\mathrm{ScS}$ measurements) were used in this travel-time plot. Even after application of the station corrections, the data show substantial scatter for all three types of arrivals. Theoretical travel-time curves for the PREM and SYL1 (Fig. 1) models are also shown. The smooth PREM model predicts only two arrivals, direct $\mathrm{S}$ and $\mathrm{ScS}$, while the discontinuity in model SYL1 produces a triplication in the $\mathrm{S}$ travel-time branch. It is not possible to say which of the two models is superior based on the fit to the $\mathrm{S}$ and $\mathrm{ScS}$ arrivals alone. The $\mathrm{ScS}$ branch for SYL1 is about a second slower than for PREM due to the negative gradient beneath the discontinuity, but this time difference is too small to be resolved by the scattered arrival time data. The existence of the intermediate arrivals clearly favors

TABLE II

Focal mechanisms for profiled events

\begin{tabular}{|c|c|c|c|c|c|}
\hline Data & & Strike & Dip & Rake & Reference \\
\hline $13 \mathrm{Apr}$. & 1969 & 274.0 & 036.0 & 090.0 & Cardwell and Isacks (1978) \\
\hline 13 Feb. & 1970 & 260.0 & 070.0 & 088.0 & Newly determined \\
\hline 04 Nov. & 1972 & 252.0 & 082.0 & 220.0 & Newly determined \\
\hline 17 May & 1974 & 270.0 & 030.0 & 035.0 & Newly determined \\
\hline 07 May & 1979 & 164.0 & 082.0 & 032.0 & Giardini (1984) \\
\hline
\end{tabular}




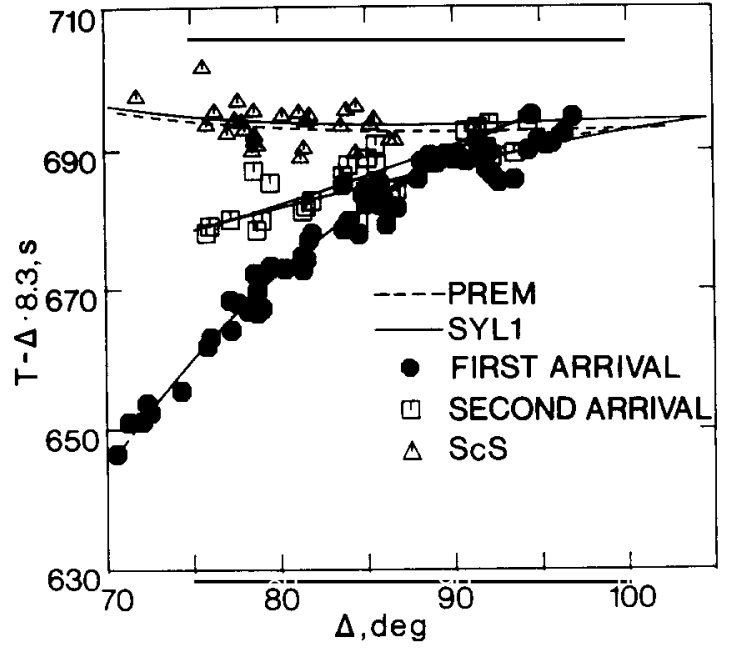

Fig. 3. Travel-time plot for combined data from all 11 events. Times and distances have been adjusted to a surface focus by ray tracing from the source depths. Second arrivals and ScS arrivals are shifted by the same amount as first arrivals. Surface depth travel-time curves for PREM and SYL1 are plotted for reference.

the discontinuity model, but accurate measurement of their timing is difficult due to interference effects in the long-period waveforms, and can only be made in limited ranges $\left(75-80^{\circ}\right.$ and $\left.92-95^{\circ}\right)$ where the arrivals are well-isolated. The intermediate arrivals also show a large amount of scatter, but are distributed around the back branches of the SYL1 travel-time curve. To better evaluate the significance of these intermediate arrivals, we need to perform an extensive waveform modeling analysis.

\section{Waveform modeling}

Lay and Helmberger (1983) demonstrated that for their data a triplication due to a lower mantle shear velocity discontinuity could account for an observed arrival intermediate to $\mathrm{SH}$ and $\mathrm{ScSH}$ in the distance range $70-82^{\circ}$ as well as for distortions of the $\mathrm{SH}$ waveforms beyond $85^{\circ}$. In this study similar comparisons were made of data profiles for the Indonesian events with synthetics for a variety of lower mantle models. Figure 4 compares an SH data profile for the 5/17/74 event (depth, $d=126 \mathrm{~km}$ ) with a synthetic profile for

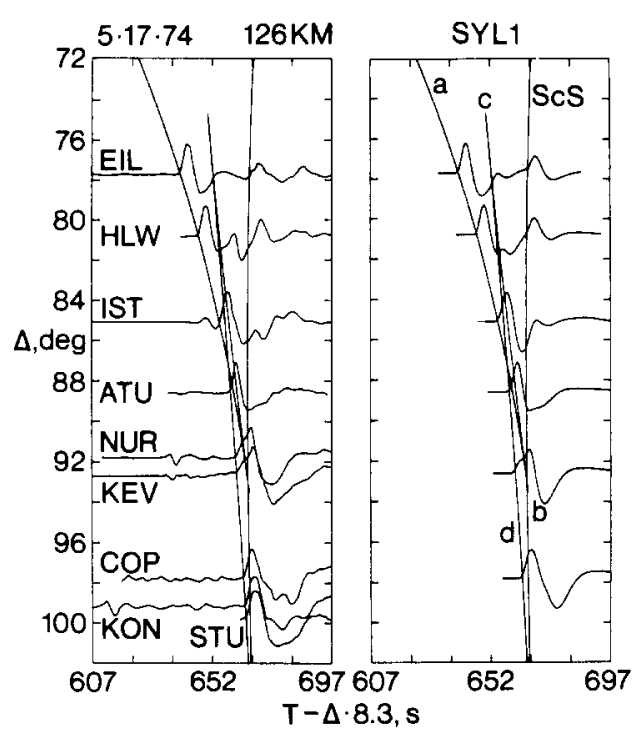

Fig. 4. Observed and synthetic profiles of long-period seismograms for the 17 May 1974 event. Travel-time curves are for model SYL1 (source depth of $126 \mathrm{~km}$ ). Amplitudes are normalized and data have been shifted to line up on first arrival branch of the travel-time curve.

our preferred average model SYL1. Synthetic waveforms were calculated using generalized ray theory with a simple point source trapezoid (total duration $=3 \mathrm{~s}$ ), a $t^{*}$ of $3.5 \mathrm{~s}$, and a long-period WWSSN instrument response. The first arrivals of the data have been aligned on the first arrival of the travel-time curve, which was calculated for model SYL1, to suppress receiver anomalies. As expected on the basis of Fig. 3, the differential times between $\mathbf{S}$ and $\mathrm{ScS}$ are generally matched well by model SYL1. The overall fits between the data and the synthetic waveforms are also quite good. The poorest fit is for HLW, where the intermediate arrival in the data is significantly larger and later than in the synthetic. It shall be shown later that the enhanced amplitude and delayed time of this arrival are characteristic of HLW, but cannot be explained by receiver structure. The ScS arrival is also later than predicted, which suggests that the intermediate arrival has a deep mantle origin. The variations in $\mathrm{SH}$ and $\mathrm{ScSH}$ amplitudes throughout the profile are adequately matched by the synthetics, which were created using the appropriate focal mechanism (Table II).

The progression of observed waveforms in Fig. 
4 can be explained by the SYL1 travel-time curve and synthetics. At distances from 72 to $75^{\circ}$ (station EIL) the cd branch of the travel-time curve, which represents energy penetrating below the discontinuity, arrives intermediate to $\mathrm{SH}$ and $\mathrm{ScSH}$, but the arrival is weak because most of the energy turns above the discontinuity (and consequently arrives as the ab branch). As distance increases (HLW, IST) the cd arrival becomes stronger and moves closer to the $a b$ arrival. The cd arrival moves through the ab arrival as the branches cross over (at ATU) and a very impulsive high frequency arrival is observed. The $\mathrm{ScS}$ arrival is fairly small at this distance because of its greater geometric spreading. Beyond crossover, the cd arrival precedes both the ScS and ab arrivals (NUR, KEV) and eventually becomes the larger arrival (COP, KON, STU), as most of the energy received at greater distances turns below the discontinuity. The broadened waveforms observed near $92^{\circ}$ are particularly important since the pulse is strongly distorted from the characteristic waveshape at both closer (EIL) and larger (STU) distances.

While it is possible to propose alternative explanations for some of the waveforms in this profile, a travel-time triplication due to a $\mathrm{D}^{\prime \prime}$ discontinuity is a straightforward explanation for the complete progression of waveforms. Some minor contamination by SKS can be seen in the tangential component profile (preceding $\mathrm{SH}$ on the KON, KEV, NUR, and IST records), but for most of the stations SKS arrives well ahead of SH and so cannot be proposed as an explanation for the waveform distortions. SKS arrives intermediate to SH and ScSH at HLW and contamination could account for some component of the anomalously large amplitude of the intermediate arrival. However, it would be necessary to have an off-azimuth rotation of nearly $40^{\circ}$ to leak a significant amount of SKS onto the SH component. Such a large rotation would lead to abnormal SH and ScSH waveforms, which are not observed. We conclude that while some SKS contamination may occur, it is not significant and cannot account for the $\mathrm{SH}$ waveforms in this profile (for a more detailed discussion of the problem of SKS contamination of SH data see Lay and Young (1986)).
It is also likely that multipathing due to the subducted slab near the source could affect this data set. Silver and Chan (1987) showed that multipathing from subduction events could cause a broadening of $\mathrm{SH}$ waveforms at stations along azimuths near to the strike of the subducted slab. Most of the stations used in this study lie along such azimuths, but the dominant SH broadening effect in our data profiles is related to distance rather than azimuth. Therefore, while slab multipathing may occur, it probably represents a small overprint on the more dominant waveform distortions arising from deep mantle structure.

The data and synthetic profiles for the 5/07/79 event $(d=106 \mathrm{~km})$ are shown in Fig. 5. This is a slightly shallower event, but the progression of waveforms is much the same as in Fig. 4 . The triplication shifts to greater distances as the source depth decreases, but the relative timing of the branches changes very little. The fit between data and synthetics for this event is also fairly good, although the timing of the intermediate arrivals in the waveforms at HLW and JER are not fit well. HLW has nearly the same waveform as the $5 / 17 / 74$ event, with a strong intermediate arrival. JER has two intermediate arrivals; however, analysis of other data from JER indicates that this

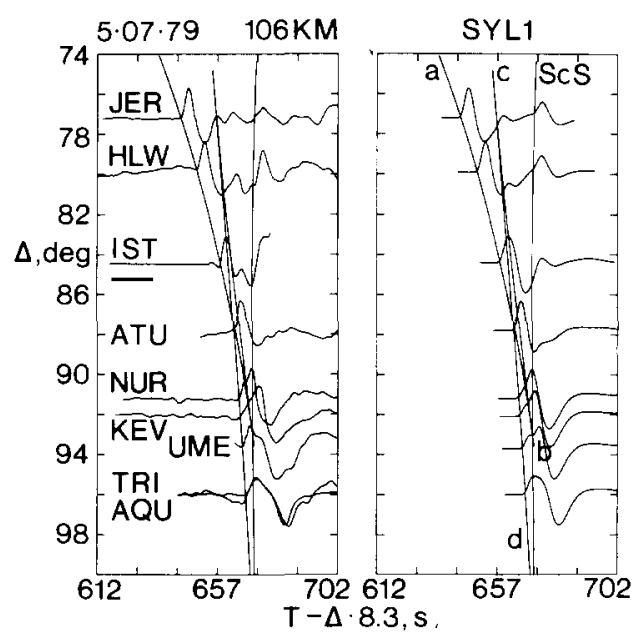

Fig. 5. Observed and synthetic profiles of long-period seismograms for the 7 May 1979 event. Travel-time curves are for model SYL1 (source depth of $106 \mathrm{~km}$ ). Amplitudes are normalized and data have been shifted to line up on first arrival branch of the travel-time curve. 
waveform is somewhat anomalous. If the cd branch were delayed slightly relative to the $a b$ branch the synthetics would match both JER and HLW better. Note that the data have been shifted to align the first arrivals on the ab branch, so such small differential time variations are reasonable. The waveform at IST, which was cutoff at the edge of a record, is also not fit particularly well by the synthetic, with its smaller time separation between the $\mathrm{ab}$ and cd arrivals. However, the observed $\mathrm{ScS}-\mathrm{Scd}$ time is well predicted by the SYL1 travel-time curve, and we suggest that heterogeneity along the ab path has caused Sab to arrive a few seconds early. The SH waveforms at the crossover distance of the ab and cd branches (ATU), and beyond are fit well by the synthetics. The negative gradient below the discontinuity in the SYL1 model leads to synthetics which fit data at diffracted distances $\left(>95^{\circ}\right)$ well, as can be seen in this and the previous profile (Fig. 4). A more thorough discussion of the modeling of data at diffracted distances is given later.

Figure 6 shows direct comparisons of data and SYL1 synthetics for several stations from the $5 / 17 / 74$ and $5 / 07 / 79$ events. The similarity of the HLW signals for the two events is evident. There may be an intermediate arrival of the size and timing shown on the synthetics for the HLW records, but it is obscured by a much larger arrival about $2 \mathrm{~s}$ later. Because we were not able to match the complete waveshape at HLW with synthetics for any reasonable lower mantle structure, even allowing for some travel-time variations, it is possible that some of this complexity may be due to SKS contamination. Another possibility is that the Sab amplitude is anomalously low due to defocussing effects, giving the appearance of a disproportionately strong second arrival. The other waveforms shown are from the crossover distance and beyond, and indicate that SYL1 matches the tremendous variation in waveshapes for this range. A model with a smooth $\mathrm{D}^{\prime \prime}$ velocity structure, such as PREM, would not fit all of these waveforms. PREM cannot account for the interference waveforms at KEV and COP for the 1974 event or at NUR and TRI for the 1979 event. It is clear that there are some features in these waveforms that are not predicted by the discontinuity model,

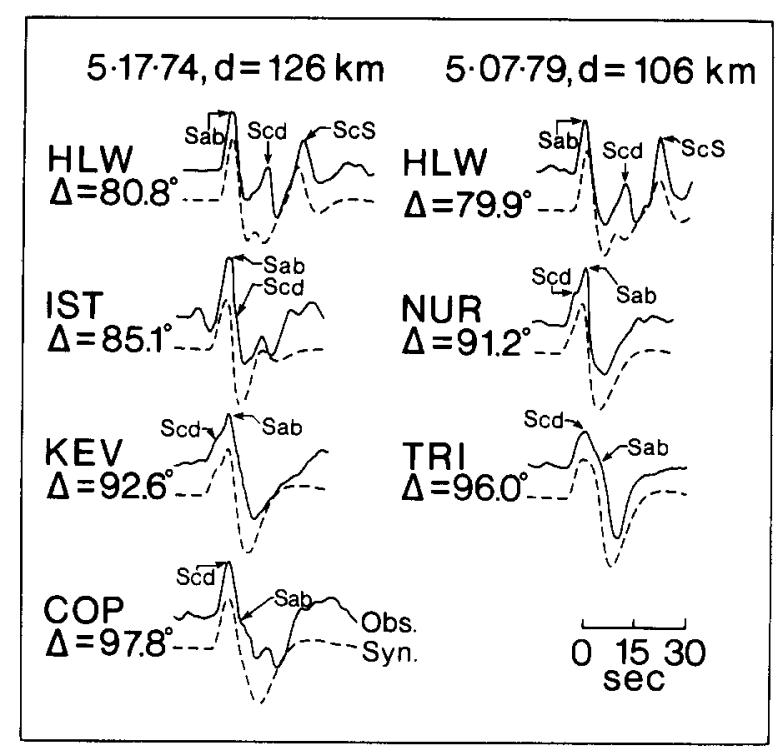

Fig. 6. Comparison of selected data and synthetic seismograms for the 17 May 1974 and 7 May 1979 events. Amplitudes are normalized. Arrows indicate arrivals of Sab, Scd and ScS phases.

which may be due to either source or receiver complexity. It is particularly important to establish the receiver effects.

To investigate the effects of structure near the stations on the SH waveforms, profiles of all recordings at each station from the Indonesian events were analyzed (Fig. 7). In these station profiles, the data have been shifted to the appropriate times and distances for a $600 \mathrm{~km}$ deep source, and a corresponding travel-time curve for SYL1 has been superimposed. If an arrival intermediate to $\mathrm{SH}$ and $\mathrm{ScSH}$ or an anomalous pulse broadening at a given station is caused by heterogeneous receiver structure then it is reasonable to assume, given the proximity in source locations for these events, that a similar effect should be seen in every signal at the same time: that is, waveform features due to receiver structure should not show significant moveout relative to the first SH arrival over these limited distance ranges.

The NAI profile in Fig. 7 does not show any features which are particularly suggestive of heterogeneity near the station. Because station NAI is close to the source area, most of the data are from distances where arrivals due to the back 

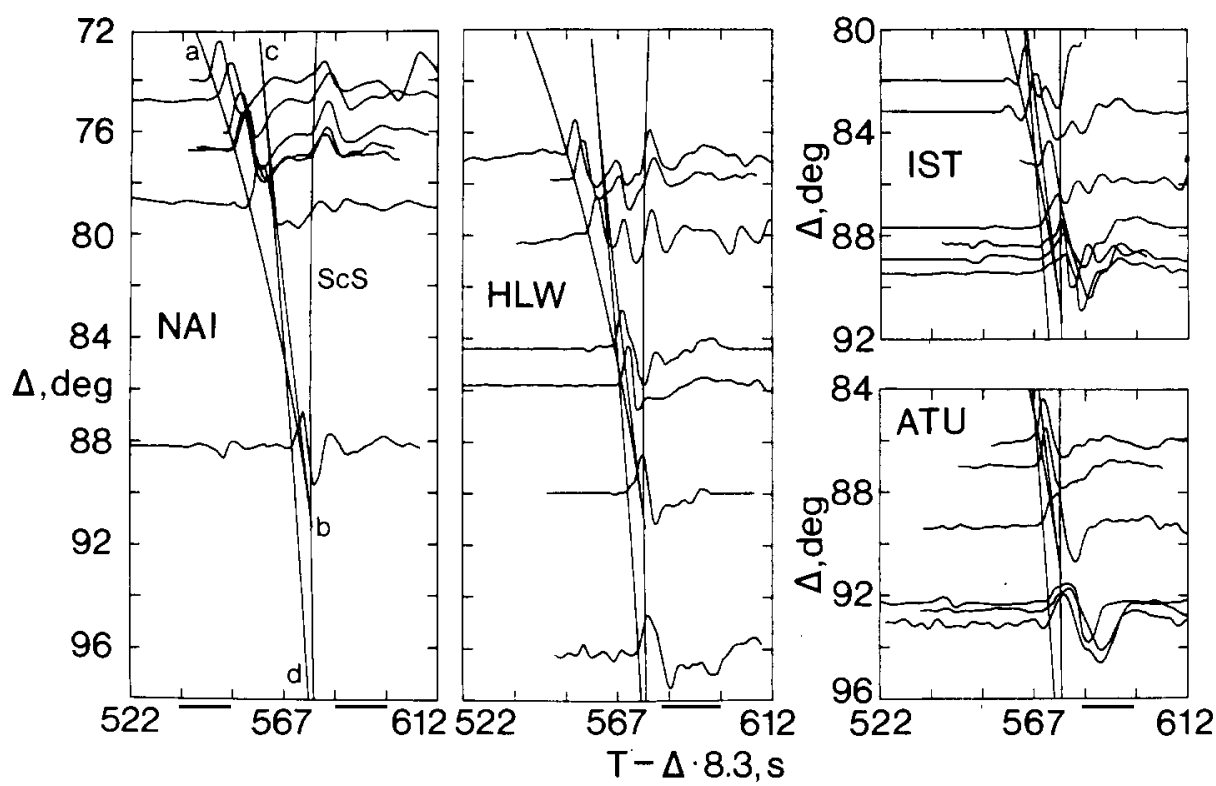

Fig. 7. Individual station profiles for the combined data set. All data have been adjusted to a common source depth of $600 \mathrm{~km}$ based on ScS-S times. Amplitudes are normalized and seismograms have been shifted to line up on superimposed SYL1 travel-time curves (source depth of $600 \mathrm{~km}$ ).

branch (cd) of the triplication would be expected to be weak. There may be a weak intermediate arrival in the NAI waveforms from 72 to $76^{\circ}$, but these waveforms could be adequately modeled with the smooth PREM model as well. The waveforms from 77 to $88^{\circ}$ show additional complexity that is consistent with SYL1, but are also located at distances that are not particularly diagnostic for proving or disproving the model.

The profile for station HLW includes the two similar records from the 5/07/79 and 5/17/74 events at about 77 and $78^{\circ}$, respectively. It is apparent from the rest of the profile that the large arrival between $\mathrm{SH}$ and $\mathrm{ScSH}$ cannot be caused by near-receiver structure because it shows moveout relative to Sab. Although the intermediate arrival is very large and is late relative to $S a b$ at closer distances, the moveout of this feature and the progression of waveforms in the HLW profile are predicted well by the SYL1 travel-time curve. The intermediate arrival moves closer to the first $\mathrm{SH}$ arrival as distance increases and the two arrivals interfere at $84^{\circ}$ and beyond. This can be explained by the crossing over of the $\mathrm{cd}$ and $\mathrm{ab}$ branches of the triplication.
The Middle Eastern station IST and the European station ATU are further from the sources, so their profiles start at greater distances. Both profiles clearly show the progression of waveforms that SYL1 would predict and which cannot be matched with the PREM model. Many of the waveforms for these two stations have broad, asymmetric upswings which might seem to be receiver related, but both profiles also have waveforms with very narrow upswings at distances corresponding to the crossover of the SYL1 travel-time curve. The progression of SH waveforms in the IST and ATU profiles from narrow to broadened upswings with increasing distance cannot be explained by receiver reverberations, but it is consistent with the crossing over and separation of the cd and ab branches of the SYL1 triplication. These station profiles provide convincing evidence that systematic receiver structure effects are not responsible for the waveform characteristics that model SYL1 matches.

Surface reflected phases that sample the deep mantle should also show triplication features if a discontinuity is present. Figure 8 shows $\mathbf{S}$ and $\mathbf{s S}$ travel-time curves calculated for shallow and deep 


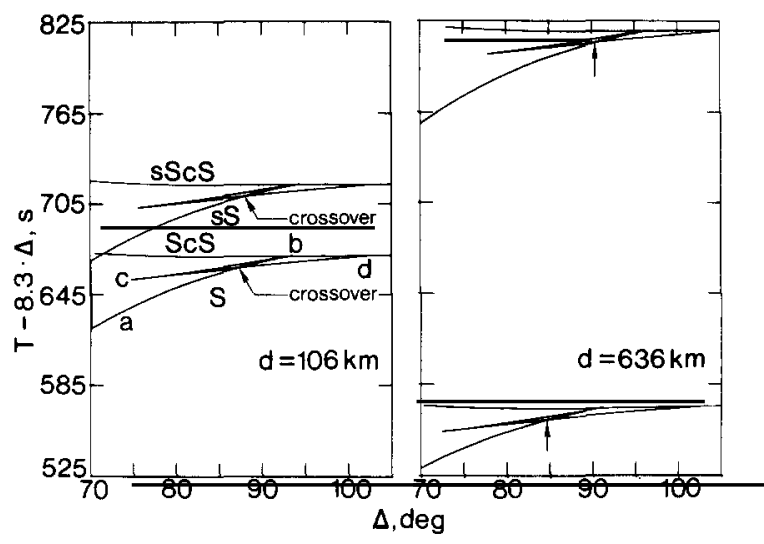

Fig. 8. $\mathrm{S}$ and sS travel-time curves, calculated using model SYL1, for shallow and deep source events. Arrows indicate the crossover points (simultaneous Sab and Scd arrivals).

events using the SYL1 model. The geometry of the sS triplications is nearly identical to that of the $S$ triplications, with the exception that the sS triplications are shifted to greater distances and times because of the extra path lengths traversed by the surface reflected phases. The crossover distance shift between the $\mathrm{S}$ and $\mathrm{sS}$ branches is directly related to the depth of the event: the deeper the event the greater the relative shift. Thus, if there is a discontinuity in $\mathrm{D}^{\prime \prime}$ the $\mathrm{SH}$ and $\mathrm{SSH}$ waveforms at any given station should be similar for a shallow event and much different for a deep event. The combination of triplication waveforms in $\mathrm{SH}$ and $\mathrm{sSH}$ is particularly strong evidence for a deep mantle interpretation of the waveform complexity (see Lay (1987) for a detailed discussion).

Figure 9 shows $\mathrm{SH}$ and $\mathrm{sSH}$ data profiles for the $4 / 13 / 69$ event $(d=152 \mathrm{~km})$. The sS phases arrive about $70 \mathrm{~s}$ after the $S$ phases. Because this is a relatively shallow event, the crossover distance shift for the sS travel-time curve is small (approximately $1^{\circ}$ ), and, thus, the SYL1 model predicts very little difference in the $\mathrm{SH}$ and $\mathrm{sSH}$ waveforms seen at each station. The $\mathrm{sSH}$ waveforms have reversed polarity due to the radiation pattern, but are otherwise nearly identical to the corresponding SH waveforms. The difference in take-off direction for $\mathrm{S}$ and sS ensures that these two phases represent different portions of the radiation pattern and have different near-source

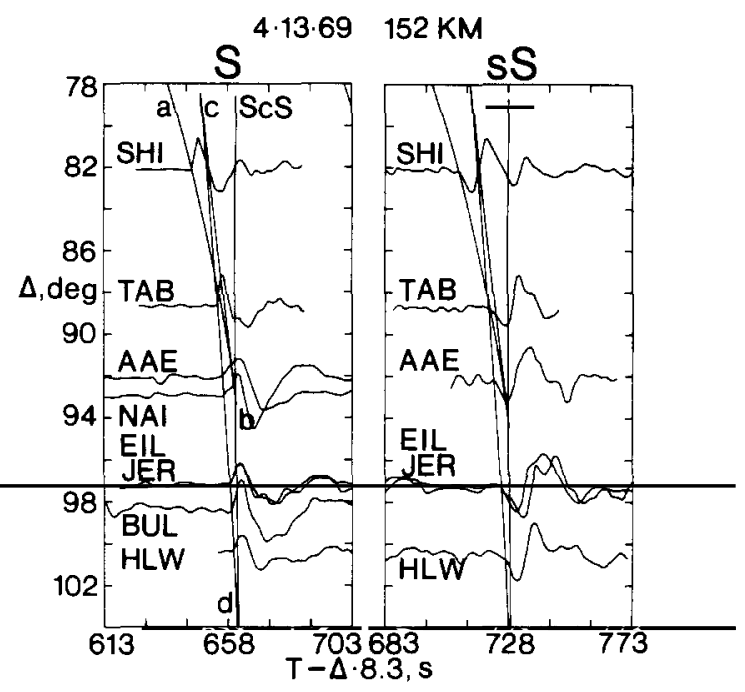

Fig. 9. $\mathrm{S}$ and sS data profiles for the 13 April 1969 event with SYL1 travel-time curves calculated (source depth of $152 \mathrm{~km}$ ): amplitudes are normalized and seismograms have been shifted to line up on first arrival branches of travel-time curves.

paths through the Earth. It follows that if some of the features in the SH waveforms that we have attributed to a triplication were instead due to complexities in the radiation patterns of the sources or to multipathing near the subducted slab, then it is unlikely that $\mathrm{SSH}$ waveforms would show the same features. However, these profiles show quite similar progressions of triplication waveforms from just before (station TAB) to well after the crossover distance; therefore, these profiles indicate that near source effects cannot account for the observed SH waveforms.

Figure 10 shows $\mathrm{SH}$ and $\mathrm{sSH}$ profiles for a much deeper event on $2 / 13 / 70(d=636 \mathrm{~km})$. The great depth of this event results in substantial additional path length for the sS phases, so the travel-time lag relative to direct $S(\sim 250 \mathrm{~s})$ and crossover distance shift $\left(\sim 5^{\circ}\right)$ of the sS traveltime curve relative to the $\mathrm{S}$ curve are large. Consequently, if model SYL1 is valid, the SSH waveforms at each station should be much different than the SH waveforms. Again allowing for the change in polarity due to the difference in takeoff angle, it can be seen that the sSH waveforms are markedly different from the SH waveforms and these differences vary systematically in a manner 


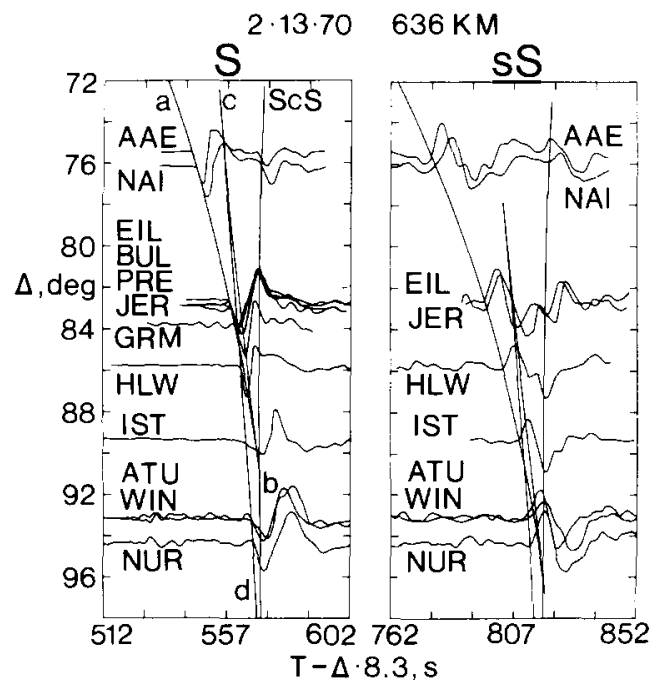

Fig. 10. S and sS data profiles for the 13 Feb. 1970 event with SYL1 travel-time curves (source depth of $636 \mathrm{~km}$ ). Amplitudes are normalized and seismograms have been shifted to line up on first arrival branches of SYL1 travel-time curves.

that is predicted by the SYL1 travel-time curves. Stations EIL, BUL, PRE, JER, and GRM are near the crossover distance of the triplication for the direct $\mathrm{S}$ phase, and thus have simple, narrow SH upswings. The sSH waveforms at the same stations correspond to a distance $5^{\circ}$ back from the crossover distance in the sS travel-time curve, where the branches are separated by several seconds, and should show distinct sSab and sScd arrivals. These strong intermediate arrivals are clearly apparent at EIL and JER. Conversely, the stations at distances beyond crossover for direct $S$ (IST, ATU, WIN, NUR) have broad SH waveforms because the cd branch has crossed in front of the ab branch, but their sSH upswings are narrow due to the $5^{\circ}$ shift for the sS waveforms that places them near the $\mathrm{sS}$ crossover distance.

A detailed comparison of $\mathrm{SH}$ and $\mathrm{sSH}$ waveforms at several of the stations for the $2 / 13 / 70$ event is shown in Fig. 11, along with synthetics for model SYL1. The sSH synthetics were created in the same way as the SH synthetics, except that a $t^{*}$ of $7.5 \mathrm{~s}$ was used to match the more attenuated sSH waveform at NUR. Station EIL is at a distance just before crossover for SH so Sab should arrive slightly ahead of Scd, creating a broadened

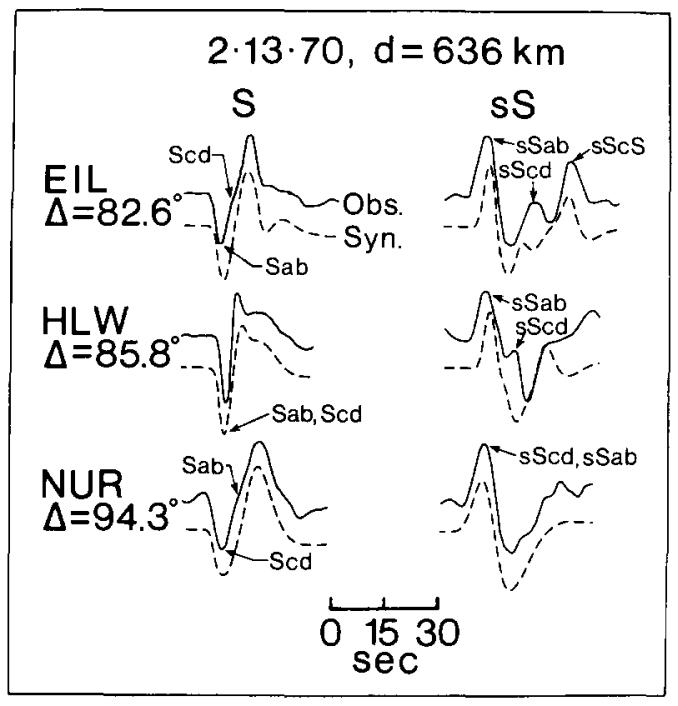

Fig. 11. Comparison of selected $\mathrm{S}$ and sS data with synthetic seismograms for the 13 February 1970 event. Amplitudes are normalized. Arrows indicate arrivals of $a b$, cd and core reflected phases.

waveform. The data in fact show a shoulder that is approximately matched by the slightly broadened SYL1 synthetic. The SSH recording for EIL clearly shows two arrivals ahead of $\mathrm{sScS}$ with a time separation of $15 \mathrm{~s}$. This waveform, which looks very much like the HLW SH recordings for the 1974 and 1979 events seen earlier (Fig. 6), can be explained by the separation of the $a b$ and $c d$ branches due to the $5^{\circ}$ shift of the sS triplication. The sSH synthetic for EIL predicts a cd arrival that is a few seconds too early, but this is an acceptable fit given the travel time and attenuation uncertainty for the sS path which may encounter subducting slab velocity heterogeneity not accounted for in the synthetic. Station HLW is located near the crossover distance for the $\mathrm{S}$ triplication so both the data and synthetic show simple impulsive waveshapes with no separation of the $a b$ and $\mathrm{cd}$ branches. The $5^{\circ}$ shift for the $\mathrm{sSH}$ waveform separates the branches and results in clearly isolated $\mathrm{ab}$ and cd arrivals. The SYL1 synthetic for SH at HLW is a good fit but the sSH synthetic does not predict a large enough separation of the branches. In spite of this, it is clear that the increased time separation of the $a b$ and $c d$ 
branches of the sS triplication relative to those of the $\mathbf{S}$ triplication offers an adequate qualitative explanation for the $\mathrm{SH}$ and $\mathrm{sSH}$ waveforms at HLW. Station NUR is $\sim 8.5^{\circ}$ beyond the SYL1 crossover and shows an $\mathrm{SH}$ waveform wherein Scd is the dominant arrival and Sab produces a slight shoulder. The synthetic matches the data quite well. Because the $5^{\circ}$ shift for the $\mathrm{sSH}$ arrival locates it just past the crossover distance, the NUR sSH waveform should have a simple narrow upswing (the upswing at crossover for SSH should be slightly broader than for $\mathrm{SH}$ due to the greater attenuation of the surface reflected path). Again, the synthetic matches the data quite well. The first order differences in $\mathrm{SH}$ and $\mathrm{sSH}$ waveforms at each station for this deep event are further evidence indicating that the waveform features, which we have attributed to a deep mantle discontinuity, cannot be explained as receiver effects, since the $S$ and sS phases at the same station should encounter similar receiver effects.

\section{Analysis of diffracted $S$ wave signals}

The Lay and Helmberger (1983) S velocity discontinuity model has been criticized (Schlittenhardt et al., 1985) because it does not match some $\mathrm{SH}$ waveforms at diffracted distances as well as a smooth model, such as JB. This difficulty can be resolved by the new shear velocity model presented here. Figure 12 shows profiles of SH synthetics for three models: JB, SLHO (Lay and Helmberger, 1983), and SYL1. The synthetics were created using the reflectivity method to ensure accuracy at diffracted distances. In the distance range $70-85^{\circ}$ an intermediate arrival is predicted by SLHO and SYL1 but not by JB. As seen in this paper and in Lay and Helmberger (1983), the data in this distance range clearly favor the discontinuity type models. At distances $>85^{\circ}$, the JB profile shows a simple arrival with a fairly symmetric and increasingly broadened upswing, whereas the SLHO profile shows a sequence of interference waveforms due to the increasing separation of the $\mathrm{ab}$ and cd branches of the triplication. The arrows indicate the post-critical Sab arrival, which should be seen in diffracted SH data if the SLHO model

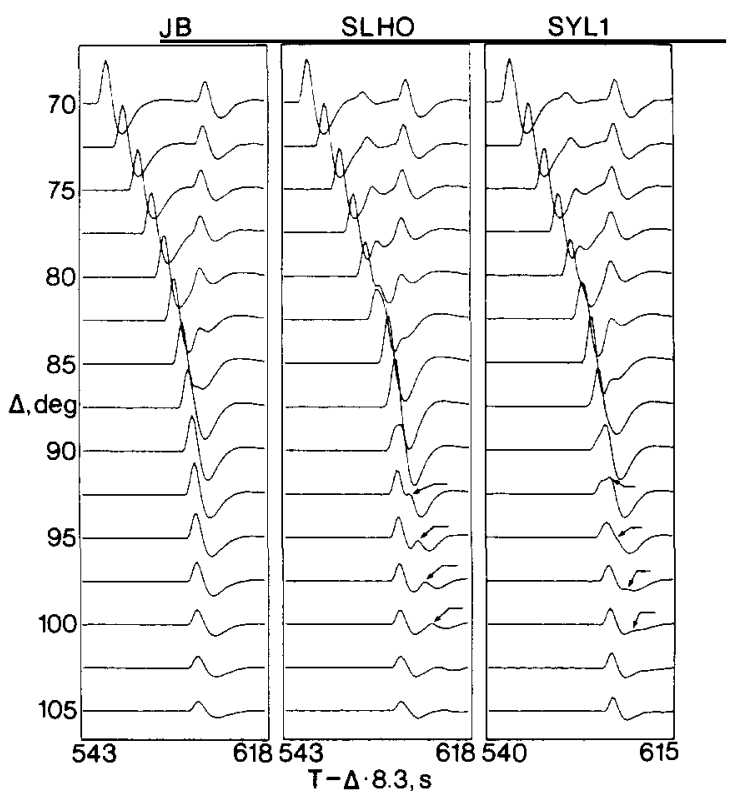

Fig. 12. Synthetic profiles of tangential component SH waves for the JB, SLHO and SYL1 models. Synthetics were computed using the reflectivity method with a source depth of 580 $\mathrm{km}$.

is correct. Such a strong arrival was not observed in the data presented by Schlittenhardt et al. (1985), who concluded that data at diffracted distances are fit better by a smooth PREM or JB model than by SLHO. They argued that the observations at closer distances require a different explanation, but did not provide one. Note that beyond $100^{\circ}$ the JB and SLHO synthetic waveforms cannot be easily distinguished and either model would be consistent with observations, especially given the typical noise levels at these distances.

The SYL1 model can match diffracted distance SH waveforms better than the Lay and Helmberger models (1983) because it has a negative velocity gradient $\left(-0.05 \mathrm{~km} \mathrm{~s}^{-1}\right.$ per $\left.100 \mathrm{~km}\right)$ beneath the discontinuity rather than a mildly positive gradient. The effect of this negative gradient is twofold. First, it slows down the rays turning below the discontinuity (i.e. Scd) which pushes the post-crossover Scd and Sab branches closer together. Secondly, the negative gradient causes more energy to turn below the discontinuity which 
weakens the post-crossover Sab arrival. A comparison of the SLHO and SYL1 waveforms (Fig. 12) clearly shows the effects of the negative gradient: the Sab arrival at distances $>90^{\circ}$ in the SYL1 profile is both earlier and smaller than the Sab arrival for the SLHO profile. We will now show that diffracted distance $\mathrm{SH}$ waveforms are fit better by SYL1 than by JB and, thus, that SYL1 fits SH data in the entire range $70-100^{\circ}$.

Figure 13 shows profiles of data and SYL1 SH waveforms for distances corresponding to crossover and beyond for the SYL1 triplication. SYL1 travel-time curves are shown for reference. The data here are a composite of post-crossover distance data from the $2 / 13 / 70,5 / 17 / 74,1 / 1 / 77$ and $5 / 07 / 79$ events. The data profile does not show the simple progression of impulsive waveforms predicted by the JB model in Fig. 12. The waveforms near $86^{\circ}$ have simple pulses, but with increasing distance the waveforms broaden and become asymmetric, implying that there are two arrivals of unequal strength within a few seconds of each other. From 89 to $91^{\circ}$ the first arrival has a smaller amplitude, but from 93 to $98^{\circ}$ the second arrival is smaller. This waveform progression can be readily explained by the tripli-

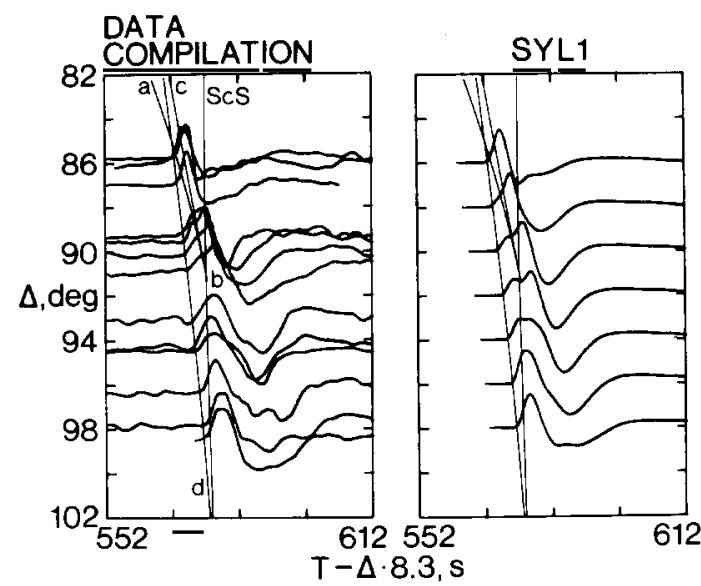

Fig. 13. Comparison of combined data (reduced to a common source depth of $600 \mathrm{~km}$ ) and SYL1 synthetics for the crossover distance and beyond. The source depth time shifts have been determined using sS-S times. SYL1 travel-time curves (source depth of $600 \mathrm{~km}$ ) are shown for reference, and the amplitudes of the data are normalized. The data have been shifted to line up on the first arrival branches of the travel-time curves. cation produced by model SYL1. Since the ab and cd branches are close together at the crossover distance, there is only one arrival. Beyond crossover, the branches begin to pull apart and the ratio of Scd to Sab amplitudes increases as more energy turns below the discontinuity. The synthetics show that the timing and relative amplitude of these effects are in agreement with the data.

\section{Discussion}

Lateral heterogeneity in the lower mantle has subtle effects on the waveform modeling used in this study, because we are intrinsically modeling differential travel times between separate branches of the travel-time curves. The region of $\mathbf{D}^{\prime \prime}$ studied here covers a range of almost $60^{\circ}$ in latitude (Fig. 2 ) and there are strong variations in upper mantle velocity structure beneath the stations and in the source region, so it is suprising how well a single radial model can match the entire data set. The scatter in the travel-time data in Fig. 3 confirms the presence of some velocity heterogeneity, but much of this appears to result from variations above the $\mathrm{D}^{\prime \prime}$ region. The $\mathrm{ScS}$ and Scd raypaths are very similar except near their deepest portions, which are both below the discontinuity; thus ScS-Scd residuals should be primarily sensitive to heterogeneity within $D^{\prime \prime}$. The Sab raypath, which turns above the discontinuity, samples different regions of the upper and lower mantles; hence, ScS-Sab and Scd-Sab residuals may be affected by heterogeneity throughout the mantle. Figure 14 shows a data profile with a superimposed SYL1 travel-time curve for the $11 / 04 / 72$ event $(d=126$ $\mathrm{km})$. The seismograms have been aligned on the $\mathrm{ScS}$ travel-time branch. The Scd and ScS branches of the SYL1 travel-time curve fit the timing of the intermediate and ScS arrivals well, but the first arrival times vary about the predicted Sab branch. If the profile is lined up on first arrivals, neither the Scd nor the ScS arrivals are predicted well. We conclude that this profile can be fit well by model SYL1 if we allow for heterogeneity along the Sab raypath. This holds true for many of the waveforms for other events, and was apparent in the study by Lay and Helmberger (1983) as well. 


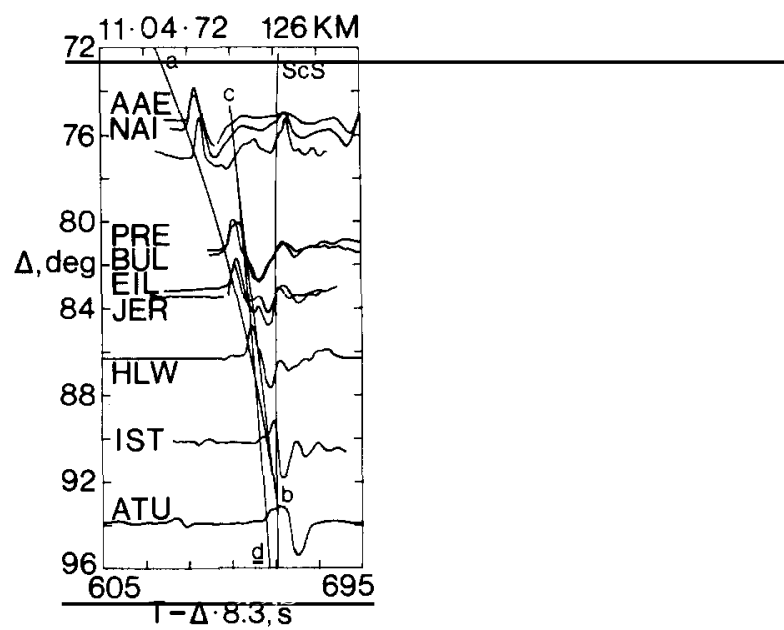

Fig. 14. Data profiles for the 4 November 1972 event with a SYL1 travel-time curve (source depth of $126 \mathrm{~km}$ ) superimposed for reference. The seismograms have been shifted to line up on ScS, and the amplitudes are normalized.

An intrinsic problem in this forward modeling approach to the determination of deep Earth structure is that the models are non-unique: there is a trade-off between the size, depth, sharpness, and velocity gradients above and below the discontinuity. Formal waveform inversion for the structure is greatly complicated by the scatter of $\mathrm{Sab}, \mathrm{Scd}$ and ScS travel times observed in the data, and we are currently developing new inversion procedures to accomodate these effects. The SYL1 model represents a simple modification of the PREM model which fits the data set; however, not all features of this model are well constrained. It was found that the data could be fit nearly as well with a model in which the velocity 'discontinuity' was spread out over $100 \mathrm{~km}$ (Fig. 15). Distributing the velocity increase requires it to be centered at a slightly shallower depth than the sharp discontinuity in order to match the travel times. The waveforms predicted at different distances along the travel-time curves for the sharp and distributed discontinuity are quite similar. The transition zone thickness cannot be tightly constrained using long-period data, but velocity increases distributed over depth ranges of more than $150 \mathrm{~km}$ can be ruled out on the basis of the waveform modeling. We inspected the correspond- ing short-period recordings for these events, but found themr to be quite poor. Hight noise levels prevent ready identification of the triplication arrivals at closer distances, where the waveforms are more sensitive to the transition zone thickness. The short-period data do not appear to be inconsistent with a discontinuity, but they do not unambiguously require a sharp velocity increase. In spite of the limited resolution of the modeling, we believe that the midpoint of the velocity increase is reliably determined to $\pm 25 \mathrm{~km}$ and the overall size of the increase to $\pm 0.25 \%$. The strength of the negative velocity gradient above the CMB is only slightly dependent on how the velocity increase is modeled, while the decrease in velocity gradient above the discontinuity is poorly constrained in all cases, being largely controlled by the choice of depth at which the reference model begins to be perturbed.

It is clearly of great importance to also address the nature of the $P$ velocity structure near the top of $\mathrm{D}^{\prime \prime}$. The source-receiver configuration used in this study was not suitable to study $P$ waves because the focal mechanisms which produce stable SH radiation to the stations tend to produce weak $P$ radiation at the same azimuths. However, the array analysis by Wright et al. (1985) sampled the $P$ velocity structure of $D^{\prime \prime}$ to the east of the region sampled by our $\mathrm{S}$ data, and also proposed a $\mathrm{D}^{\prime \prime}$ discontinuity. Although the $\mathrm{P}$ velocity discontinuity model of Wright et al. (1985) is similar in general character to the $S$ velocity discontinuity model SYL1, the two models are not completely compatible. The $\mathbf{P}$ velocity model has a similar size discontinuity $(2.5-3.0 \%)$ and a negative velocity gradient beneath it, but the $\mathbf{P}$ discontinuity is located $\sim 100 \mathrm{~km}$ deeper than the SYL1 S discontinuity. The Wright et al. (1985) $P$ wave model is based on a sharp change in $\mathrm{P}$ slowness at $87^{\circ}$, which they attributed to the crossing over of the branches of a triplication caused by a deep mantle discontinuity.

It is important to consider the effects of such a large $P$ velocity discontinuity, since Schlittenhardt (1984) has argued that the expected secondary arrivals at pre-crossover distances are not observed in $P$ wave data recorded by the Graefenberg array. A change in slowness of the first arrival can 

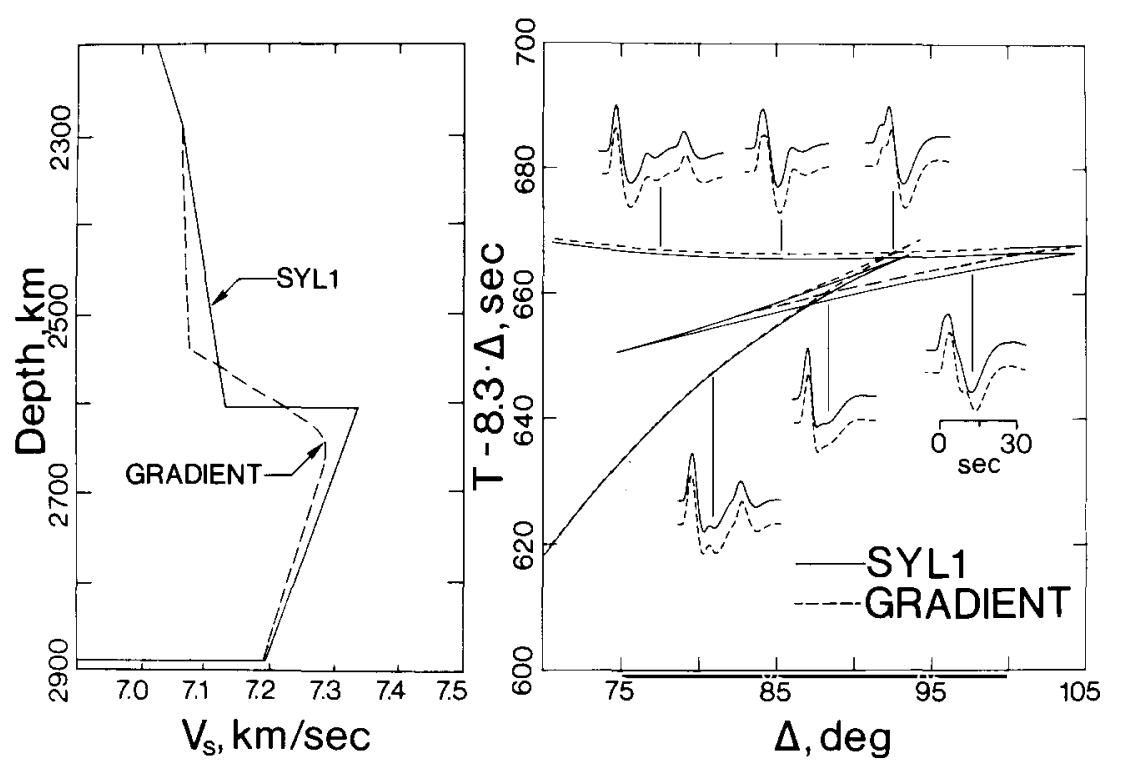

Fig. 15. (Left) Velocity vs. depth profiles for SYL1 and an alternative gradient model with a distributed velocity increase. (Right) Travel-time curves for both models are shown along with comparisons of synthetic seismograms calculated using generalized ray theory with a source depth of $126 \mathrm{~km}$.

also be explained by a distributed velocity increase rather than a discontinuity, since either type of structure will cause a triplication in the travel-time curve. Figure 16 shows profiles of

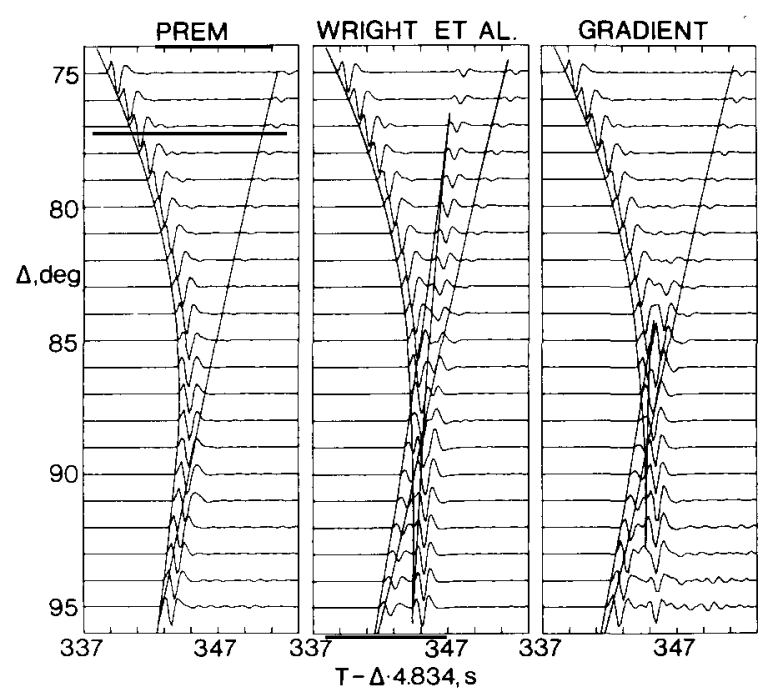

Fig. 16. Synthetic profiles for short-period $P$ waves along with corresponding travel-time curves for the PREM, Wright et al. (1985) and a gradient modification of the Wright et al. models. The source depth is $0 \mathrm{~km}$. short-period $\mathrm{P}$ wave synthetics computed for PREM, the Wright et al. (1985) model, and a P velocity model with the same size velocity increase distributed over $100 \mathrm{~km}$. The synthetics were calculated using generalized ray theory with a simple point source, a $t^{*}$ of $1.5 \mathrm{~s}$, and a WWSSN short-period instrument response. We did not include a focal mechanism because this is a comparison of synthetics only. In the distance range $75-87^{\circ}$, the Wright et al. (1985) model predicts a strong secondary arrival which PREM does not predict. The high noise level of typical short-period $P$ coda tends to obscure this portion of the data, so it is generally difficult to detect this arrival without array processing like that used by Schlittenhardt (1984); however, such strong secondary arrivals have not been clearly identified in any study. The absence of such secondary arrivals at pre-crossover distances can be explained by the gradient model, which produces intermediate arrivals that are well below the level of detection, especially given that the short-period PcP arrival itself is seldom detected at these distances (Schlittenhardt, 1984). Long-period P waves will be less sensitive to the nature of the velocity 
increase and should show secondary arrivals of equivalent size for either type of structure, but the phases arrive so close together that they are not separated in long-period waveforms. The amplitude of the secondary arrivals in the short-period synthetics can also be decreased by reducing the size of the $\mathbf{P}$ velocity increase, although such a modification may not match the change in first arrival slope. Beyond the crossover distance $\left(88^{\circ}\right)$, both the Wright et al. model (1985) and gradient model predict distorted waveforms due to the separation of the branches of the $P$ wave triplication. It would seem possible to choose between the discontinuity/gradient models and PREM by comparing synthetics with observations at these distances, but the complexity of the signals presented by Wright et al. (1985) prevents a clear selection of a preferred model.

Diffracted long-period $\mathrm{P}$ waves for a discontinuity model similar to the $S$ wave models of Lay and Helmberger (1983) were computed by Schlittenhardt et al. (1985) and compared with observations. The data show little evidence to support such a $P$ velocity model; however, this does not rule out the possibility of other $P$ velocity discontinuity models. Undiffracted signals provide much greater sensitivity to the $D^{\prime \prime}$ structure, and further analysis of $\mathrm{P}$ data is needed, especially for distances near $90-95^{\circ}$. Given the apparent similarity and uncertainty of the proposed $\mathrm{P}$ and $\mathrm{S}$ velocity discontinuity/gradient models it would seem likely that models with more consistent features should be attainable. However, we were unable to find a pair of reasonable $\mathrm{P}$ and $\mathrm{S}$ models which would simultaneously fit our data set and the Wright et al. (1985) observations. The $100 \mathrm{~km}$ difference in depth of the velocity increases seems to be too large to attribute to uncertainty in the two data sets, unless very strong perturbations of the reference models are allowed. One could appeal to lateral heterogeneity to explain this discrepancy, since identical regions of $\mathrm{D}^{\prime \prime}$ are not sampled, but it would be more convincing to analyze $P$ and $S$ data for the same paths.

The similarity in size and depth of the S velocity discontinuity proposed here for the $\mathrm{D}^{\prime \prime}$ region beneath India and the Indian Ocean to the discontinuity models for the $\mathrm{D}^{\prime \prime}$ region beneath Alaska, the Caribbean and northern Eurasia (Fig. 1, Lay and Helmberger, 1983) suggests that the discontinuity may be a global feature. There is undoubtedly additional lateral heterogeneity superimposed on these simple models; however, we have not yet identified a region that does not show evidence for an $\mathrm{S}$ velocity discontinuity. The interpretation of this feature is not straightforward, particularly given the uncertain nature of the corresponding $P$ velocity structure. This rapid increase in velocity implies a comparable density increase, suggesting a chemical or phase change at the top of $\mathrm{D}^{\prime \prime}$. A reasonable interpretation is that the $\mathrm{D}^{\prime \prime}$ region is a compositionally distinct layer at the base of the mantle. If so, then it is also likely that there is a thermal boundary layer at the top of $D^{\prime \prime}$, which would be consistent with the decrease in velocity gradient just above the discontinuity (Jeanloz and Richter, 1979). The negative velocity gradient beneath the discontinuity, which is required to fit the diffracted data, is generally consistent with the presence of a thermal boundary layer just above the CMB, though it is believed that this boundary layer should be $<100$ km thick (Jeanloz and Richter, 1979; Doornbos 1983).

An abrupt velocity increase at the top of the $D^{\prime \prime}$ region would probably represent a chemical change, and imply that any convection in $\mathrm{D}^{\prime \prime}$ is separate from the rest of the mantle. If the velocity increase at the top of $\mathbf{D}^{\prime \prime}$ is distributed, however, it would imply that the density increase near the top of $D^{\prime \prime}$ is continuous, and this could be interpreted as either a phase change or a gradational compositional change. In either case the $D^{\prime \prime}$ region may participate in the lower mantle convection system. This would be consistent with the model of Davies (1984), in which an increase in viscosity with depth causes heterogeneities, some of which may represent material subducted from the upper mantle (Hoffmann and White, 1982), to be concentrated in the lowermost mantle. A rapid increase in the concentration of heterogeneities near the top of $D^{\prime \prime}$ could account for the velocity increase in the 'discontinuity' models. In this type of model, $\mathrm{D}^{\prime \prime}$ would not be a separate convective system, but materials would move through it slowly due to the high viscosities. Sufficiently high viscos- 
ities could lead to residence times of billions of years, providing a reservoir of primordial material which could satisfy geochemical isotopic observations. Entrainment of some of the heterogeneities into the convective processes may account for observations of larger scale heterogeneity in $\mathrm{D}^{\prime \prime}$, while small scale thermal and compositional fluctuations would explain the shorter wavelength heterogeneity in the region. Further work on the global extent, sharpness, and correspondence between $\mathrm{P}$ and $\mathrm{S}$ velocity discontinuities in $\mathrm{D}^{\prime \prime}$ is required before definitive statements about $D^{\prime \prime}$ composition and dynamics can be made.

\section{Conclusions}

The SH and sSH waveforms from African, Middle Eastern and European stations for events in the Java Trench-Timor Trough are consistent with a velocity model with an abrupt $2.75 \% \mathrm{~S}$ velocity increase $\sim 280 \mathrm{~km}$ above the core-mantle boundary and a negative velocity gradient $(-0.05$ $\mathrm{km} \mathrm{s}^{-1}$ per $100 \mathrm{~km}$ ) between the discontinuity and the core. Alternative explanations of the waveform complexity, such as SKS contamination, source complexity, multipathing, or receiver reverberations, cannot account for the observed systematic changes in the waveforms with distance. The size and depth of the inferred discontinuity are consistent with the models that Lay and Helmberger (1983) proposed for regions of the deep mantle beneath Alaska, the Caribbean, and Eurasia, which suggests that the sharp increase in shear velocity near the top of $\mathrm{D}^{\prime \prime}$ may be a global feature. The negative velocity gradient in $D^{\prime \prime}$, which is necessary to fit waveforms at distances from 90 to $100^{\circ}$ that are not fit well by the Lay and Helmberger models, represents a significant modification of those models. However, it is possible to fit the original Lay and Helmberger data sets using discontinuity models with negative velocity gradients as well (Lay 1985), eliminating any inconsistency with diffracted $\mathrm{S}$ waveshapes. Hence, the negative velocity gradient within the $\mathrm{D}^{\prime \prime}$ region, which is consistent with a thermal boundary layer above the CMB, may be global as well.

\section{Acknowledgments}

Most of the seismic records were obtained from the Lamont Doherty Geological Observatory filmchip collection. We thank Lisa Donaghe, Chris Lynnes, and Susan Schwartz for reviewing the manuscript and providing helpful comments. C.J. Young was supported by an NSF Graduate Fellowship. This research was supported by NSF Grants EAR-8407792, EAR-8451715, a Shell Faculty Career Initiation Grant, and the Alfred P. Sloan Foundation.

\section{References}

Alexander, S.S. and Phinney, R.A., 1966. A study of the core-mantle boundary using $P$ waves diffracted by the Earth's core. J. Geophys. Res., 71: 5943-5958.

Bolt, B.A. and Niazi, M., 1984. S velocities in $D^{\prime \prime}$ from diffracted SH-waves at the core boundary. Geophys. J.R. Astron. Soc., 79: 825-834.

Bullen, K.E., 1949. Compressibility-pressure hypothesis and the Earth's interior. Mon. Not. R. Astron. Soc., 5: 355-368.

Cardwell, R.K. and Isacks, B.L., 1978. Geometry of the subducted lithosphere beneath the Banda Sea in eastern Indonesia from seismicity and fault plane solutions. J. Geophys. Res., 83: 2825-2838.

Clayton, R.W. and Comer, R.P., 1983. A tomographic analysis of mantle heterogeneities from body wave travel time data. EOS, 64: 776.

Cleary, J.R. and Haddon, R.A.W., 1972. Seismic wave scattering near the core-mantle boundary: a new interpretation of precursors to PKIKP. Nature, 240: 549-551.

Davies, G.F., 1984. Geophysical and isotopic constraints on mantle convection: an interim synthesis. J. Geophys. Res., 89: $6017-6040$.

Dillinger, W.H., Harding, S.T. and Pope, A.J., 1972. Determining maximum likelihood body wave focal plane solutions. Geophys. J.R. Astron. Soc., 30: 315-329.

Doornbos, D.J., 1983. Present seismic evidence for a boundary layer at the base of the mantle. J. Geophys. Res., 88: 3498-3505.

Doornbos, D.J. and Vlaar, N.J., 1973. Regions of seismic wave scattering in the Earth's mantle and precursors to PKP. Nature Phys. Sci., 243: 58-61.

Dziewonski, A.M., 1984. Mapping the lower mantle: determination of lateral heterogeneity in $\mathbf{P}$ velocity up to degree and order 6. J. Geophys. Res., 89: 5929-5952.

Dziewonski, A.M. and Anderson, D.L., 1981. Preliminary reference Earth model. Phys. Earth Planet. Inter., 25: 297-356.

Dziewonski, A.M., Hager, B.H. and O'Connell, R.J., 1977. Large-scale heterogeneities in the lower mantle. J. Geophys. Res., 82: 239-255. 
Giardini, D., 1984. Systematic analysis of deep seismicity: 200 centroid moment tensor solutions for earthquakes between 1977 and 1980. Geophys. J.R. Astron. Soc., 77: 883-914.

Gutenberg, B. and Richter, C.F., 1939. On seismic waves. Beitr. Geophys., 54: 94-136.

Haddon, R.A.W. and Cleary, J.R., 1974. Evidence for scattering of seismic PKP waves near the mantle-core boundary. Phys. Earth Planet. Inter., 8: 211-234.

Helmberger, D.V., 1974. Generalized ray theory for shear dislocations. Bull. Seismol. Soc. Am., 64: 45-64.

Hofmann, A.W. and White, W.M., 1982. Mantle plumes from ancient oceanic crust. Earth Planet. Sci. Lett., 57: 421-436.

Husebye, E.S., King, D.W. and Haddon, R.A.W., 1976. Precursors to PKIKP and seismic wave scattering near the mantle-core boundary. J. Geophys. Res., 81: 1870-1882.

Jeanloz, R. and Richter, F.M., 1979. Convection, composition, and the thermal state of the lower mantle. J. Geophys. Res., 84: $5497-5504$.

Jeffreys, H., 1939. The times of P, S, and SKS and the velocities of $P$ and S. Mon. Not. R. Astron. Soc., 4: 498-533.

King, D.W., Haddon, R.A.W. and Cleary, J.R., 1974. Array analysis of precursors to PKIKP in the distance range 128 to $142^{\circ}$. Geophys. J.R. Astron. Soc., 37: 157-173.

Lay, T., 1985. Analysis of diffracted $\mathbf{S}$ waves traversing a region with a lower mantle shear velocity discontinuity. EOS, 66: 310 .

Lay, T., 1986. Evidence for a lower mantle shear velocity discontinuity in S and sS phases. Geophys. Res. Lett., 13: 1493-1496.

Lay, T. and Helmberger, D.V., 1983. A lower mantle S-wave triplication and the shear velocity structure of $D^{\prime \prime}$. Geophys. J.R. Astron. Soc., 75: 799-838.

Lay, T. and Young, C.J., 1986. The effect of SKS scattering on models of the shear velocity structure of the $\mathrm{D}^{\prime \prime}$ region. $\mathrm{J}$. Geophys., 59: 11-15.

Schlittenhardt, J., 1984. Array-Untersuchungen von reflektierten und diffraktierten Kernphasen. $\mathrm{PhD}$ thesis, University of Frankfurt.

Schlittenhardt, J., Schweitzer, J. and Muller, G., 1985. Evidence against a discontinuity at the top of $\mathrm{D}^{\prime \prime}$. Geophys. J.R. Astron. Soc., 81: 295-306.

Sengupta, M.K. and Toksoz, M.N., 1976. Three dimensional model of seismic velocity variation in the Earth's mantle. Geophys. Res. Lett., 3: 84-86.

Sengupta, M.K., Hassell, R.E. and Ward, R.W., 1981. Threedimensional seismic velocity structure of the Earth's mantle using body wave travel times from intra-plate and deepfocus earthquakes. J. Geophys. Res., 86: 3913-3934.

Silver, P.G. and Chan, W.W., 1987. Observations of body-wave multipathing from broadband seismograms: evidence for lower-mantle slab penetration beneath the Sea of Okhotsk. J. Geophys. Res., 91: 13787-13802.

Wright, C., 1973. Array studies of $P$ phases and the structure of the $D^{\prime \prime}$ region of the mantle. J. Geophys. Res., 78: 4965-4982.

Wright, C., 1975. The origin of short period precursors to PKP. Bull. Seismol. Soc. Am., 65: 765-780.

Wright, C. and Lyons, J.A., 1975. Seismology, $d T / d \Delta$ and deep mantle convection. Geophys. J., 40: 115-138.

Wright, C. and Lyons, J.A., 1980/1981. Further evidence for radial velocity anomalies in the lower mantle. Pageoph, 119: 137-162.

Wright, C., Muirhead, K.J. and Dixon, A.E., 1985. The P wave velocity structure near the base of the mantle. J. Geophys. Res., 90: 623-634.

Young, C.J. and Lay, T., 1987. The core-mantle boundary. Ann. Rev. Earth Planet. Sci., 15: 25-46. 